\title{
Fachkräftepolitik als strategische Zukunftsaufgabe
}

\author{
WOLFGANG SCHROEDER, RALF RUKWID, JAN MACHNIG
}

\section{Einleitung}

Verschiedene Megatrends werden den deutschen Arbeitsmarkt längerfristig entscheidend formen und neue Anforderungen für dessen nachhaltige Modernisierung mit sich bringen. Eine der größten Herausforderungen der kommenden Jahre ist der demografische Wandel. Entsprechende Modellrechnungen kommen zu dem Ergebnis, dass je nach Basisannahmen das Erwerbspersonenpotenzial bereits bis 2025 um 4 bis 7 Mio. Erwerbspersonen zurückgehen wird. Die Zahl der Schulabgänger wird sinken und der Anteil älterer Erwerbspersonen steigen. Zugleich verknüpft sich mit der fortschreitenden Digitalisierung ein grundlegender Umbruch der Arbeitswelt. Mit der Entwicklung zur sogenannten Industrie 4.0 wird in der Fertigungstechnik eine neue Qualität der Informatisierung erreicht. Für die Facharbeiter bedeutet dies einen fundamentalen Wandel der Arbeitsanforderungen, bei dem u. a. das notwendige Überblicks- bzw. Vernetzungswissen stark zunehmen wird. Unter diesen sich grundlegend wandelnden demografischen und technologischen Voraussetzungen gilt es, die Verfügbarkeit von ausreichend qualifizierten Arbeitskräften in der Zukunft zu sichern. Angesichts der hohen und sich qualitativ verändernden künftigen Bedarfe dürfen die bedeutenden Fachkräftepotenziale bei den Geringqualifizierten, den Personen mit Migrationshintergrund, den älteren Erwerbspersonen und den Frauen nicht länger ungenutzt bleiben, sondern müssen durch verbesserte Ein- und Aufstiegsstrategien gezielt erschlossen werden. Fachkräfte sind und bleiben der Motor von Innovationen und damit der Garant für einen zukunftsfähigen und modernen Industriestandort.

Von Arbeitgeberseite wird immer wieder auf die Notwendigkeit hingewiesen, einem wachsenden Fachkräftemangel entgegenzuwirken. So betonte etwa Arbeitgeberpräsident Ingo Kramer gegenüber der dpa: „Wenn wir nicht gegensteuern, werden die volkswirtschaftliche Leistungskraft und unsere Sozialsysteme massiv gefährdet ". ${ }^{1}$ Für die Sicherung des Fachkräftebedarfs fordern die Arbeitgeber u. a. eine Verlängerung der Lebensarbeitszeit, eine konsequente Umsetzung der Rente mit 67 und eine Erhöhung der (wöchentlichen) Arbeitszeit. Zugleich setzen sie auf eine qualifizierte Zuwanderung. Paradoxerweise sinkt jedoch in Deutschland die Ausbildungsbereitschaft der Unternehmen - trotz der Klagen über Stellenbesetzungsprobleme und zu- nehmende Fachkräfteengpässe. Inzwischen bildet nur noch rund jeder fünfte Betrieb überhaupt aus. Bei der Anzahl der neu abgeschlossenen Ausbildungsverträge wurde nun schon wiederholt ein historischer Tiefstand erreicht. In den Metallund Elektroberufen ist die Zahl der Jung-Facharbeiter seit 1994 um 36,5\% zurückgegangen. Von den Unternehmen als den zentralen Akteuren der Fachkräftesicherung wäre jedoch ein angemessenes Engagement in der Ausbildung zu erwarten. Angesichts der weitreichenden technologischen und demografischen Veränderungen muss neben den Arbeitgebern auch die Politik im Sinne der künftigen Fachkräftesicherung rechtzeitig und vorsorgend handeln und ihre Gestaltungsspielräume nutzen. Handlungsbedarf besteht auf mehreren Politikfeldern wie der Bildungs-, Arbeitsmarkt-, Sozial- und Familienpolitik. Die politischen Maßnahmen müssen dabei mit der tarif- und betriebspolitischen Praxis für alle Beschäftigten verknüpft werden und den spezifischen Kontexten von Regionen, Branchen und Berufsprofilen Rechnung tragen.

Für eine langfristig erfolgreiche Fachkräftesicherung schlägt die IG Metall dementsprechend ein umfassendes Programm mit aufeinander abgestimmten Instrumenten und Maßnahmen auf vier zentralen Handlungsfeldern vor: gute Bildung und Qualifizierung, gute Arbeit und mehr und bessere Aufstiegsmöglichkeiten, eine bessere Verzahnung von Beruf und Privatleben sowie gute Arbeitsbeziehungen (Übersicht 1).

\section{Fachkräftesicherung braucht gute Bildung und bessere Qualifizierung}

Mit dem Wandel der Wirtschaftsstrukturen hin zu wissensintensiven Industrien und Dienstleistungen ist Bildung mehr denn je der Schlüssel für Fortschritt, Wachstum und Innovationen sowie für die individuellen Emanzipationsund Entwicklungschancen. Wer den Bedarf an Fachkräften sichern will, muss das Bildungs- und Ausbildungssystem sanieren. Das aktuelle Bildungssystem wird den unterschiedlichen Anforderungen der Megatrends Digitalisie-

1 Vgl. u.a. http://www.wiwo.de/erfolg/beruf/fortschrittsbericht2014-dem-fachkraeftemangel-ist-nicht-beizukommen/ 11356910.html. 
rung, Höherqualifizierung und Flexibilisierung nicht gerecht. Die Zahl der Ungelernten in Deutschland ist nach wie vor signifikant hoch. Wie viele Studien belegen, ist das deutsche Bildungssystem im internationalen Vergleich zudem hoch selektiv. Die Bildungs- und Erwerbsbiografien sind maßgeblich durch die soziale Herkunft und den familiären Hintergrund vorbestimmt. Höhere Investitionen in Ganztagesangebote und in sonstige Verbesserungen von Vorschulen sowie allgemeinbildenden und berufsbildenden Schulen sind notwendig, um herkunftsunabhängige Bildungschancen zu verwirklichen. Im Mittelpunkt der Forderungen der IG Metall steht „eine gute Schule für alle“, in der alle Kinder bis zum 10. Schuljahr gemeinsam unterrichtet und individuell gefördert werden. Pädagogisch wie ökonomisch besonders sinnvoll ist dabei eine spezielle Förderung von Kindern und Jugendlichen mit einer relativ schlechten Ausgangsposition.

Angesichts der dynamischen Anforderungen an die Erwerbstätigen ist die Beruflichkeit zu erweitern und zu modernisieren. Der Erwerb beruflicher Qualifikationen ist eine wichtige Basis für die langfristige Sicherung einer anspruchsvollen und gut bezahlten Tätigkeit sowie den Erwerb weiterer beruflicher Kompetenzen. Die betrieblich duale und die hochschulische Berufsbildung sind in diesem $\mathrm{Zu}$ sammenhang als gleichwertig zu betrachten. Die IG Metall setzt sich dafür ein, dass sich diese Gleichwertigkeit auch in der betrieblichen Praxis und im Bildungssystem konkret abbildet. Die betriebliche Personalpolitik ist auf eine Offenheit der Fach- und Führungskarrieren für Beschäftigte mit unterschiedlichen Bildungswegen auszurichten. Vor dem Hintergrund der in den Studien und Projektionen des Bundesinstituts für Berufsbildung (BBIB) und des Instituts für Arbeitsmarkt- und Berufsforschung (IAB) ${ }^{2}$ festgestellten drohenden Engpässe auf der Ebene der beruflich qualifizierten Fachkräfte muss die Bedeutung und Attraktivität der beruflichen Bildung insbesondere auch für die leistungsstärkeren Schüler wieder deutlich aufgewertet werden. Der bisherige deutsche Sonderweg, demzufolge in der Regel zwischen der betrieblichen und akademischen Ausbildung unterschieden wird, bedarf hierbei einer Anpassung, um die immer noch stark eingeschränkte Durchlässigkeit zwischen diesen beiden Teilbereichen zu verbessern. Eine stärkere Öffnung des Hochschulzugangs für Berufserfahrene ohne Abitur, eine Ausweitung von Stipendienprogrammen sowie eine Freistellung junger Beschäftigter zum Studium mit Rückkehroption könnten einen wichtigen Beitrag leisten. Die hochschulischen Bildungsprozesse sollten sich in bestimmten Fachbereichen zudem stärker an dem Konzept der Beruflichkeit orientieren, um die Studierenden besser auf das Arbeitsleben außerhalb der Hochschule vorzubereiten.

Trotz einer robusten Konjunktur wurde im Jahr 2014 ein historischer Tiefstand bei den neuen Ausbildungsverträgen erreicht. Immer weniger Betriebe bilden noch aus, und vor allem die kleineren Unternehmen ziehen sich zunehmend aus der dualen Ausbildung zurück. Die IG Metall
ÜBERSICHT

\section{Vier zentrale Handlungsfelder zur Fachkräftesicherung*}

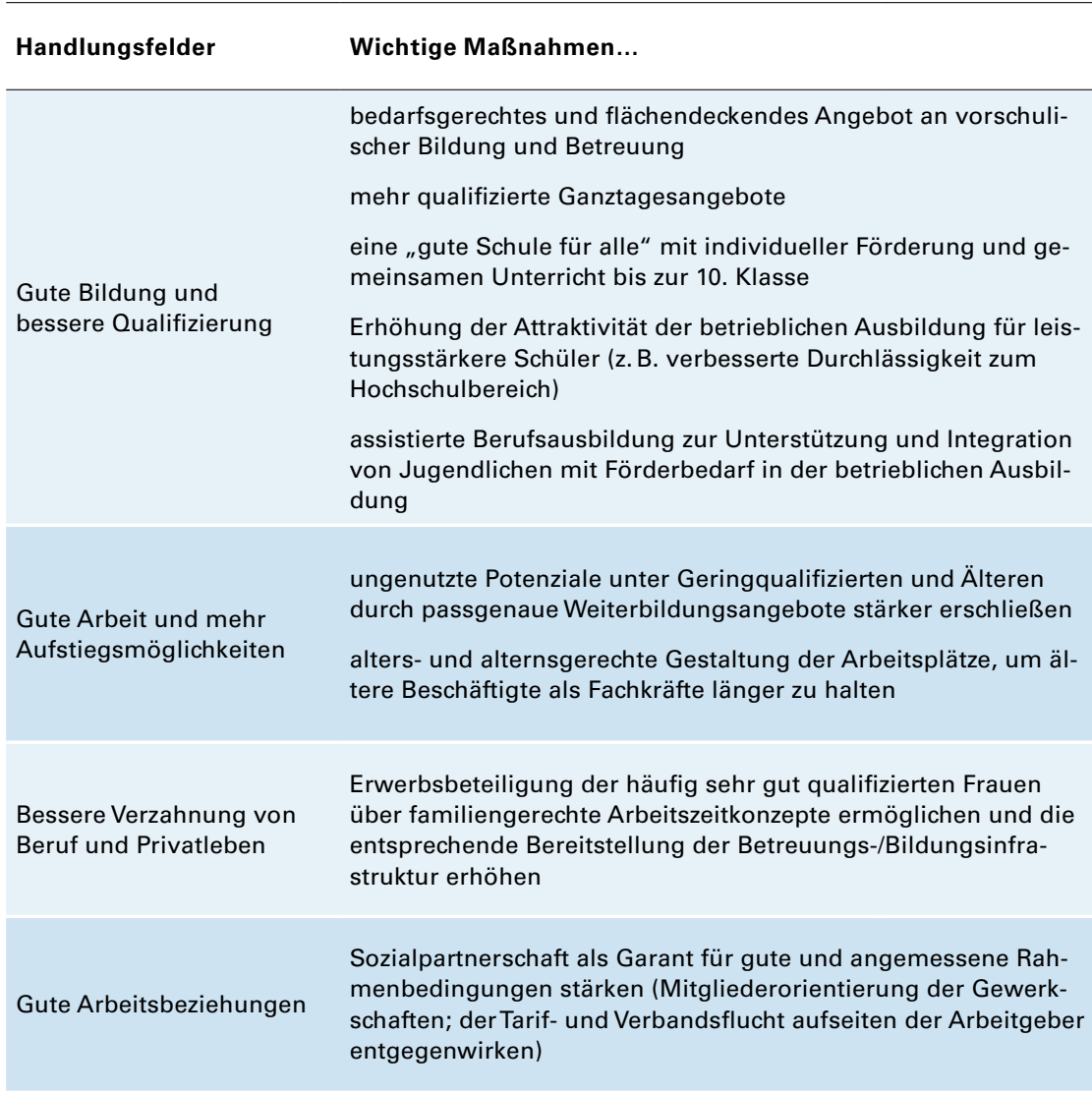

"Anm.: Die als Beispiel genannten Einzelmaßnahmen sind teils an mehrere Handlungsfelder andockbar. Die hier gewählte Zuordnung folgt dem Grundsatz der Priorität.

Quelle: Darstellung der Autoren.

fordert die Arbeitgeber daher auf, ihre Ausbildungsbemühungen wieder zu verstärken. Die in Zukunft drastisch sinkende Zahl der Schulabgänger erfordert eine grundlegende Neuausrichtung der bisherigen Ausbildungsstrategien. Statt einer vermeintlichen Bestenauslese gilt es, Jugendliche mit sehr unterschiedlichen Begabungen und Fähigkeiten besser in die betriebliche Berufsausbildung zu integrieren. Für Jugendliche mit Hauptschulabschluss müssen - anstelle von Warteschleifen in einem zunehmend als reformbedürftig angesehenen Übergangssektor - wieder mehr Ausbildungsplätze angeboten werden. Sinnvoll ist darüber hinaus eine verstärkte Ausbildung von jungen Frauen im technischen Bereich und eine verbesserte Unterstützung von Ju-

2 Vgl. Helmrich, R./Zika, G./Kalinowski, M./Wolter, M. I. (2012): Engpässe auf dem Arbeitsmarkt: Geändertes Bildungs- und Erwerbsverhalten mildert Fachkräftemangel. Neue Ergebnisse der BIBB-IAB-Qualifikations- und Berufsfeldprojektionen bis zum Jahr 2030: Bundesinstitut für Berufsbildung, BIBB Report 18/12, Bonn. 
gendlichen mit individuellen Förderbedarfen (assistierte Berufsausbildung).

Für eine dauerhafte Sicherung des Fachkräftebedarfs muss die berufliche Fort- und Weiterbildung zum integralen Bestandteil der Unternehmenspolitik werden. Der Erhalt und die Weiterentwicklung beruflicher Konsequenzen gewinnen für die Beschäftigten an Bedeutung, da sich mit Digitalisierung und technologischem Wandel die Anforderungsprofile ständig ändern. Wichtig sind hierbei lernförderliche Arbeitsstrukturen, die einen niedrigschwelligen Zugang zu Kompetenzen und anerkannten Zertifikaten befördern. Beim Zugang zu beruflicher Weiterbildung zeigen sich jedoch nach wie vor Ungleichheiten anhand von Qualifikation, Erwerbsstatus oder Migrationshintergrund. Die vorhandenen Qualifizierungstarifverträge müssen als Grundlage für eine systematische Qualifizierungspolitik der Unternehmen besser genutzt werden. Die IG Metall hat sich in den tarifpolitischen Auseinandersetzungen zudem für das Instrument der Qualifizierungs-Teilzeit eingesetzt. Eine solche Teilzeitregelung eröffnet auch den Ungelernten, die bisher am wenigsten an Weiterbildungsmaßnahmen teilhaben konnten, wichtige berufliche Qualifizierungsmöglichkeiten.

\section{Fachkräftesicherung braucht gute Arbeit sowie mehr und bessere Aufstiegsmöglichkeiten}

Anhand der traditionellen Arbeitsmarktindikatoren, wie Arbeitslosigkeit und Beschäftigung, zeigt sich eine positive Entwicklung in Deutschland. Die rein statistische Entwicklung der Erwerbstätigkeit sagt aber wenig über die Art und Qualität der konkreten Arbeitsverhältnisse aus und darüber, ob die Menschen davon „gut" leben können. Tatsächlich ist der deutsche Arbeitsmarkt inzwischen tief gespalten. Bereits seit Mitte der 1990er Jahre nimmt die Bedeutung des Normalarbeitsverhältnisses ab. Immer mehr Erwerbspersonen müssen sich mit atypischen Beschäftigungsverhältnissen begnügen und sind mit geringfügiger Beschäftigung, Leiharbeit oder Befristungen konfrontiert. Parallel kam es zu einer deutlichen Zunahme von Niedriglohnarbeit. Diese gilt unter vielen Politikern und der Mehrheit der Ökonomen als wichtige Beschäftigungsoption für gering qualifizierte Arbeitskräfte mit einer vermeintlich niedrigen Produktivität. Tatsächlich besitzen jedoch rund drei von vier Beschäftigten im deutschen Niedriglohnsektor einen Ausbildungsoder Hochschulabschluss. Für diese qualifizierten Arbeitskräfte ist der Niedriglohnbereich zur beruflichen Sackgasse geworden.

Vor dem Hintergrund der Klagen über eine zunehmende Knappheit an Fachkräften erscheint die Ausweitung von atypischen und teils prekären Beschäftigungsverhältnissen paradox. Statt die Beschäftigten zu fördern, werden vorhandene Potenziale nicht genutzt, Qualifikationen entwertet und die Aufwärtsmobilität ausgebremst. Zugleich wird Arbeit von den Unternehmen seit Jahren verdichtet, entgrenzt und intensiviert. Die Beschäftigten sollen immer mehr leisten und über die reguläre Arbeitszeit hinaus verfügbar sein. Dass dadurch große physische und psychische Belastungen entstehen, hat auch die Beschäftigtenbefragung der IG Metall bestätigt. Der räuberische Umgang mit der Arbeitskraft geht zulasten der Gesundheit und führt maßgeblich dazu, dass viele Beschäftigte weit vor dem eigentlichen Rentenalter aus dem Erwerbsleben ausscheiden müssen.

Prekarisierung und gesundheitlich belastende Arbeitsbedingungen sind mit dem Ziel der Fachkräftesicherung unvereinbar. Mit dem Konzept „gute Arbeit“ will die IG Metall zu einer Überwindung der tiefen Spaltung auf dem Arbeitsmarkt beitragen. Gute Arbeit wird angemessen bezahlt, ist unbefristet und bietet stabile Arbeitsverhältnisse. Sie fordert und füllt die Menschen aus, ohne sie unnötig zu belasten. Die Erfahrungen in Ostdeutschland zeigen, dass ohne gute Arbeit und deren tarifliche Absicherung die Gefahr einer Abwanderung qualifizierter Arbeitskräfte groß ist. Über die Einführung eines gesetzlichen Mindestlohns hinaus gilt es daher, den Arbeitsmarkt grundlegend neu zu ordnen. Die IG Metall fordert u. a. eine Abschaffung sachgrundloser Befristungen, Equal-Pay für Leiharbeitnehmer, ein Ende des Missbrauchs von Werkverträgen und eine gleichberechtigte Gestaltung guter und angemessener Arbeitsbedingungen für alle Beschäftigten. Um die Gesundheit, die Leistungsfähigkeit und die Motivation der Beschäftigten langfristig zu sichern, setzt sich die IG Metall zudem für eine verbindliche Anti-Stress-Verordnung ein.

Der Blick auf die unterschiedlichen Beschäftigtengruppen bestätigt, dass vorhandene Fachkräftepotenziale stärker ausgeschöpft bzw. erschlossen werden müssen. Dies gilt zunächst für die große Gruppe der Frauen, die häufig sehr gut qualifiziert sind, deren Erwerbsquote in Deutschland aber geringer ausfällt als etwa in den skandinavischen oder angelsächsischen Ländern. Auch die älter werdenden Belegschaften stellen die Unternehmen vor neue Herausforderungen. Wie die IG Metall-Beschäftigtenumfrage belegt, liegen eine alters- und alternsgerechte Arbeitswelt und Personalpolitik noch in weiter Ferne. Insgesamt glauben nur $4 \%$ der Befragten, dass ihr Betrieb gut auf älter werdende Belegschaften vorbereitet ist. Um die Erfahrungen und das Fachwissen älterer Beschäftigter langfristig zu sichern, besteht dringender Handlungsbedarf. Wichtige Maßnahmen wären z. B. eine präventive Gesundheitsvorsorge, eine ergonomische Gestaltung des Arbeitsumfelds, altersgemischte Teams, passgenaue Weiterbildungsangebote und angemessene Arbeitszeitmodelle.

Besonders benachteiligten Personengruppen sollte auch besonders geholfen werden. Personen mit Migrationshintergrund sind in der dualen Berufsausbildung stark unterrepräsentiert. Nur wenn es gelingt, diese Benachteiligungen aufzuheben, kann ihr Fachkräftepotenzial umfassend und nachhaltig aktiviert werden. Um Zugewanderte besser in den Arbeitsmarkt zu integrieren, bedarf es klarer Rahmenbedingungen bezüglich der Anerkennung ausländischer Berufsabschlüsse und gezielter Qualifizierungsmaßnahmen. 
Gering qualifizierte Personen können sich berufliche Weiterbildung oftmals nicht leisten bzw. bekommen in ihren Unternehmen keine Chance, an solchen Maßnahmen teilzunehmen. Notwendig sind hier mehr und besser zugeschnittene Qualifizierungsangebote, die letztlich zu vollwertigen beruflichen Abschlüssen führen. Entsprechend gilt es, auch den Instrumentenkasten der aktiven Arbeitsmarktpolitik auf abschlussorientierte Aufstiegsqualifizierungen in Verbindung mit einer guten und zielgruppenorientierten Beratung auszurichten. Arbeitsmarktpolitisch ist darüber hinaus eine Neujustierung der Arbeitsvermittlung geboten. Diese muss angesichts zunehmender Fachkräfteengpässe wieder stärker die vorhandenen Qualifikationen der Bewerber berücksichtigen. Die bisherigen Zumutbarkeitsregelungen sowie der Vorrang einer schnellen Vermittlung vor der aktiven Arbeitsförderung sind zu revidieren. Die Workfare-Strategie muss durch eine vorsorgende Sozialpolitik ersetzt werden. Und die setzt auf Qualifizierung.

\section{Fachkräftesicherung braucht eine bessere Verzahnung von Beruf und Privatleben}

Die IG Metall plädiert für ein neues Normalarbeitsverhältnis, das die unterschiedlichen Bedürfnisse in den wechselnden Lebensphasen stärker im Blick hat. Arbeit, Leben, Familie und Beruf müssen besser miteinander vereinbar gemacht werden. Ein Arbeitszeitmodell für alle - das passt schon lange nicht mehr. Arbeitgeber und Politik müssen es den Beschäftigten ermöglichen, auf die Unwägbarkeiten des Alltags, wie etwa familiäre Pflegeverpflichtungen, flexibel zu reagieren.

Die Erhöhung des Fachkräfteangebots über eine ansteigende Frauenerwerbstätigkeit erfordert eine neue Aufteilung der Arbeit innerhalb der Familie. Viele Frauen sind unfreiwillig in Teilzeit beschäftigt oder nicht erwerbstätig, weil sich Familie und Beruf nicht gut miteinander vereinbaren lassen. Sie würden bei einer flexibleren Arbeitszeitgestaltung gerne länger arbeiten. Gefragt sind u. a. Möglichkeiten der individuellen Zeitentnahme aus Arbeitszeitkonten, kurze Vollzeitmodelle mit verbindlichen Rückkehroptionen nach beruflichen Auszeiten sowie Homeoffice-Modelle. Um die Frauenerwerbsquote zu erhöhen, bedarf es neben einer angemessenen Gestaltung der Arbeitszeiten und einer nicht-diskriminierenden Entlohnung eines Ausbaus der entsprechenden Infrastruktur (qualifizierte Kinderbetreuung, Ganztagsschulen).

\section{Fachkräftesicherung braucht gute Arbeitsbeziehungen}

Die Sozialpartnerschaft repräsentiert die traditionelle Basis des bundesdeutschen Wirtschafts- und Gesellschaftsmodells. Die sozialpartnerschaftliche Zusammenarbeit auf der Branchenebene und die Mitbestimmung auf der Betriebsebene haben sich bei der Bewältigung der jüngsten Finanz- und Wirtschaftskrise bewährt. Auch in Zukunft sollte eine moderne Sozialpartnerschaft der Garant für die Definition und Verwirklichung guter und sinnvoller Rahmenbedingungen bleiben. Die IG Metall stellt sich zur erfolgreichen Bewältigung dieser Aufgabe ihrer Verantwortung im Bereich Mitgliederorientierung und beteiligungsorientierter Konfliktlösung. Auf Arbeitgeberseite muss für eine verlässliche Sozialpartnerschaft der in den letzten 20 Jahren erfolgten Verbands- und Tarifflucht entgegengewirkt werden. Die Sicherung des Fachkräftebedarfs ist hier ein wichtiges Argument, die Anreize für eine ordentliche Verbandsmitgliedschaft wieder in den Mittelpunkt zu rücken. Tarifvertraglich geregelte Entgelt- und Arbeitsbedingungen sind eine zentrale Grundlage für die Unternehmen, um im Wettbewerb um knapper werdende Fachkräfte und Auszubildende erfolgreich zu sein.

In den Betrieben fungieren die Betriebsräte als wichtige Vermittler und als Sammelstelle von Know-how bei den für die Fachkräftesicherung relevanten Maßnahmen wie bspw. der lernförderlichen Arbeitsgestaltung. Dort, wo es betriebliche Mitbestimmung gibt, sorgt ein stabiles Betriebsklima für eine geringere Fluktuation. Dies erhöht für die Unternehmen den Anreiz zur Weiterbildung ihrer Mitarbeiter. Gute Arbeitsbeziehungen und betriebliche Mitbestimmung fördern also Innovationen und die Sicherung und Bindung von Fachkräften.

\section{Fazit}

Von den zu koordinierenden Maßnahmen sind folgende Bereiche als zentral bedeutsam hervorzuheben:

(1) Für von der sozialen Herkunft unabhängige Bildungschancen und Schulerfolge bedarf es u. a. eines bedarfsgerechten und flächendeckenden Angebots an vorschulischer Bildung und Betreuung, mehr qualifizierter Ganztagesangebote sowie nicht zuletzt einer grundlegenden Sanierung der allgemeinen schulischen Bildung. Im Mittelpunkt der Forderungen der IG Metall steht „eine gute Schule für alle“ mit einem gemeinsamen Unterricht und individueller Förderung für alle Kinder bis zum 10. Schuljahr.

(2) Die duale Berufsausbildung ist neben der Tarifautonomie und der Sozialpartnerschaft die entscheidende Grundlage für Innovationen, wirtschaftlichen Erfolg und Wachstum in Deutschland. Der rückläufigen Zahl der Ausbildungsverträge muss mit einer Erhöhung der Attraktivität der betrieblichen Ausbildung für leistungsstärkere Schüler entgegengewirkt werden (z. B. verbesserte Durchlässigkeit zum Hochschulbereich). Zugleich müssen Schulabgänger mit teils schwierigen Ausgangslagen durch Maßnahmen wie die assistierte Berufsausbildung besser unterstützt und integriert werden.

(3) Ungenutzte Potenziale unter Geringqualifizierten und Älteren müssen durch passgenaue Weiterbildungsan- 
gebote stärker erschlossen werden. Zudem sollten über die alters- und alternsgerechte Gestaltung der Arbeitsplätze die älteren Beschäftigten als Fachkräfte länger gehalten werden.

(4) Die Erwerbsbeteiligung der häufig sehr gut qualifizierten Frauen muss über familiengerechte Arbeitszeitkonzepte und eine entsprechende Bereitstellung der für die Berufsausübung notwendigen Infrastruktur nachhaltig erhöht werden.

Um die Weichen für eine zukunftssichere und wettbewerbsfähige moderne Industrie zu stellen, sind verschiedene Arenen, Instrumente und Akteure aufeinander zu beziehen, die sich wechselseitig bedingen. Deshalb plädiert die IG Metall für eine auf vier Handlungsfelder konzentrierte Strategie. Vor allem die Unternehmen stehen in der Verantwortung: auf der betrieblichen Ebene, indem sie gute Arbeit und Aufstiegsbedingungen schaffen sowie individuelle Beteiligung und kollektive Mitbestimmung ausweiten; auf der überbetrieblichen Ebene, indem sie zu einer sozialpartnerschaftlich gestalteten Selbstregulierung und zur Tarifbindung zurückkehren. Aber auch alle anderen relevanten Akteure müssen eingebunden werden, um die ungenutzten Fachkräftepotenziale zu heben. In diesem Sinne ist die hier vorgestellte Fachkräftestrategie eine Win-win-Konstellation, die zur nachhaltigen Modernisierung des deutschen $\mathrm{Ar}-$ beitsmarktes beitragen kann.

\section{AUTOREN}

WOLFGANG SCHROEDER, Prof. Dr., lehrt Politikwissenschaften an der Universität Kassel und ist Leiter der Grundsatzabteilung der IG Metall, Frankfurt a. M.

wolfgang.schroeder@uni-kassel.de

RALF RUKWID, IG Metall Vorstand, Funktionsbereich Grundsatzfragen und Gesellschaftspolitik, Ressort Grundsatzfragen. Arbeitsschwerpunkte: Arbeitsmarktpolitik und Bildungsökonomik.

ralf.rukwid@igmetall.de

JAN MACHNIG, IG Metall Vorstand, Funktionsbereich Grundsatzfragen und Gesellschaftspolitik, Ressort Grundsatzfragen. Arbeitsschwerpunkte: Arbeitsmarkt- und Sozialpolitik.

@jan.machnig@igmetall.de 